\title{
ORIGINAL
}

\section{ESTIMACIÓN DE LA PREVALENCIA DE TRASTORNOS BIPOLARES TIPO I EN ESPAÑA A TRAVÉS DEL CONSUMO DE CARBONATO DE LITIO (1996-1998)}

\author{
Juan J Criado- Álvarez (1,2), Jaime A Domper Tornil (2) y Gloria de la Rosa Rodríguez (1) \\ (1) Sección de Investigación Epidemiológica. Centro Regional de Salud Pública. Consejería de Sanidad. Junta \\ de Comunidades de Castilla- La Mancha. \\ (2) Sección de Salud Mental. Servicio de Asistencia Sanitaria. Consejería de Sanidad. Junta de Comunidades de \\ Castilla- La Mancha.
}

\section{RESUMEN}

Fundamento: Los estudios sobre la prevalencia de Trastornos Bipolares Tipo I son escasos y están realizados con metodologías diferentes, lo que impide conocer el impacto de los mismos como problema de salud pública en España. Se determina la prevalencia de Trastornos Bipolares tipo I en España a través del consumo de carbonato de litio.

Métodos: Se estima la prevalencia a través del cálculo de las dosis diarias definidas por 100.000 habitantes y día de cartonato de litio (Grupo terapéutico, N05AN), durante los años 1996-1998, para cada una de las provincias españolas. Se ha dispuesto de los datos de las prescripciones indicadas por la Subdirección General de Planificación Farmacéutica del Ministerio de Sanidad y Consumo.

Resultados: Se estima una prevalencia de 70 casos por 100.000 habitantes y día para el conjunto de la población española.

Conclusiones: El consumo de carbonato de litio por provincias y años permite estimar la distribución de los Trastornos Bipolares tipo I en España. Las cifras obtenidas sitúan a España en una prevalencia baja- media; aunque las diferencias metodologicas no permiten conclusiones definitivas.

Palabras clave: Trastomos bipolares. Litio. Farmacoepidemiología. Prevalencia. Dosis Diaria Definida.

\section{ABSTRACT
Estimate of type I bipolar disorder prevalence (1996-1998)

Background: Different methodologies have been employed for conducting the few studies made on the prevalence of Type I Bipolar Disorders, which makes it impossible to know what impact these disorders have had on public health in Spain. The prevalence of Type I Bipolar Disorders is calculated by the degree to which lithium carbonate is used.

Methods: The prevalence is estimated by calculating the daily doses of lithium carbonate prescribed per 100,000 inhabitants/day (Treatment Group, NO5AN) throughout the 1996-1998 period for each one of the provinces in Spain. The data based on the prescriptions written was provided by the Pharmaceutical Planning Bureau of the Ministry of Health and Consumer Affairs.

Results: A prevalence of 70 cases $/ 1000,000$ inhabitants/day for the overall population of Spain was estimated.

Conclusions: Lithium carbonate usage by provinces and by years affords the possibility of estimating the distribution of Type I Bipolar Disorders in Spain. The figures calculated rank Spain at a low-average prevalence, although the methodological differences prevent it from being possible to draw any final conclusions.

Key words: Bipolar disorders. Lithium. Drug epidemiology. Prevalence. Daily Dose Defined

Correspondencia:

Juan José Criado Alvarez

Cl. Santa Sabina, $30-3^{\circ} \mathrm{A}$

Talavera de la Reina

45.600 Toledo

Correo electrónico: jjcriado@jccm.es

Los resultados de este trabajo forman parte de estudios previos del «Plan de Salud Mental de Castilla- La Mancha, 1999-2003». 


\section{INTRODUCCIÓN}

Los Trastornos bipolares (TB) son una de las entidades más clásicas de la psiquiatría. Su antigua denominación de psicosis maniacodepresiva (PMD) se debe a Kraepelin, quien reunió en esta entidad los cuadros de manía y melancolía, siendo un término que ha desaparecido de la CIE-10 y del DSM-IV. En 1.975 Fieve y Dunner subdividieron los TB en bipolar tipo I (PMD maníaca o CIE-9: 296.1) y bipolar tipo II (PMD depresiva junto a episodios de hipomanía o CIE-9: 296.2). En España se han realizado investigaciones epidemiológicas comunitarias con el objeto de conocer la distribución de la patología psíquica, como el del Baix Llobregat (1.987) y el de Cantabria (1.987), utilizando una técnica de doble fase, y obteniendo una cifra de prevalencia puntual total para el tipo I de 80 casos por 100.000 habitantes $^{1,2}$.

Una forma diferente de estimar la prevalencia es a través de estudios de utilización de medicamentos. Estos se convierten en indicadores de la prevalencia de problemas médicos $\mathrm{y}$, en cierto modo, en indicadores de la solución farmacológica que adopta la comunidad médica ante los problemas de salud $^{3}$. La frecuencia de los TB puede ser conocida a través del consumo de carbonato de litio, del que solo existe una presentación comercial en España. Se trata de un fármaco de utilización terapéutica prácticamente específica en los TB, en especial en los de Tipo I, por lo que generalmente casi todos los pacientes que utilizan este fármaco padecen este tipo de trastornos, aunque no ocurre lo mismo a la inversa, es decir, no todos aquellos pacientes con este tipo de trastorno consume este fármaco. El consumo de carbonato de litio no puede considerarse un buen estimador de la prevalencia del TB tipo II, patología en la que se utilizan con frecuencia otros fármacos en la prevención y tratamiento, principalmente carbamacepina y ácido valproico ${ }^{5-8}$. El consumo del carbonato de litio ha seguido una tendencia cre- ciente de uso en España desde que se autorizó en marzo de 1970, su toma y prescripción se realiza de forma crónica y permanente, no existen formas de difícil dosificación (gotas, colirios, soluciones), no existen altibajos aparentes en su comercialización, el acceso a este fármaco es fácil y económico debido a que es de aportación reducida a la Seguridad Social, la atención comercial que recibe es prácticamente nula y su eficiencia es incuestionable ${ }^{8-11}$.

El objetivo de este trabajo es conocer el consumo de carbonato de litio en España (1996-1998), como indicador de la prevalencia de TB Tipo I. Existe un trabajo previo de los autores referido solo a la comunidad autónoma de Castilla- La Mancha ${ }^{13}$.

\section{SUJETOS Y MÉTODOS}

El presente estudio se ha realizado entre 1998 y 1999 mediante el análisis de los datos de prescripción del grupo terapéutico N05AN de la Anatomical Therapeutic Chemical (ATC) $)^{15,16}$, y en especial las de carbonato de litio, ofrecidos por la Subdirección General de Planificación Farmacéutica del Ministerio de Sanidad y Consumo, para los años 1996 a 1998, haciendo referencia a la única especialidad existente en el mercado español (Plenur $\left.{ }^{\circledR}\right)$, adquirida mediante la receta oficial del Sistema Nacional de Salud $^{10}$. El consumo obtenido por esta fuente es, habitualmente, considerado como una aproximación aceptable del consumo real ${ }^{3,4,11,17-21}$. Los datos de consumo se expresan en dosis diarias definidas (DDD) por 100.000 habitantes y día (DHD=DDD/ 100.000 habitantes/día). Se ha utilizado el valor de la DDD propuesta por la Nordic Council on Medicines, que es de $880 \mathrm{mg}^{1} 1$. La DHD (Dosis Habitante Día) estima con carácter de tasa poblacional, el número de personas que, en un momento dado, (prevalencia de punto) están consumiendo una dosis terapéutica diaria para los TB. El cálculo de la DDD por 100.000 habitantes y día (DHD) se obtiene de la fórmula: 
DHD $=(($ DDD consumidas entre 19961.998)/ (Población total * tiempo en días)) $* 100.000$

El numerador se obtiene por la suma de los $\mathrm{mg}$ del principio activo prescritos en el período 1996-1998, en la única especialidad farmacéutica del principio activo estudiado, dividida por la DDD estándar expresada en $\mathrm{mg}$. En el denominador, la población utilizada se refiere a la del Padrón de $1996^{12}$ y se considera la misma para cada año, multiplicándose ésta por el tiempo expresado en días. La DHD expresa el número de dosis promedio que se han prescrito para el consumo en un día, lo que equiparamos a las personas consumidoras de una dosis terapéutica diaria promedio por cada 100.000 habitantes. Ello nos ofrece una prevalencia de enfermos en un día del período estudiado por 100.000 habitantes, la cual es una técnica utilizada en múltiples estudios $3,4,11,17-19,22,23$.

\section{RESULTADOS}

Considerado el carbonato de litio como tratamiento específico de todos los pacientes con TB tipo I, se estima una prevalencia nacional de 63 casos por 100.000 habitantes en 1996 y de 70 casos por 100.000 habitantes en 1998 (DHD agrupada para todo el territorio nacional). Ello representa en cifras absolutas entre 24.992 y 27.768 enfermos en el período estudiado.

La tabla 1 reproduce la prescripción de carbonato de litio en DHD (Dosis Diaria Definida/ 100.000 habitantes/ Día) en el período 1996-1998 en España por provincias y año, con diferencias entre ellas, con unas mayores tasas en Navarra, Lleida, Girona, Ávila y Soria; mientras que las cifras más bajas son en Huelva, Cádiz, Sevilla, Málaga, A Coruña, Orense, Pontevedra y Valladolid.

Tabla 1

Consumo de carbonato de litio en DHD $\left({ }^{*}\right)$ por provincias y año en España (1.996-1.998)

\begin{tabular}{|lcccc|}
\hline PROVINCIA & 1.996 & 1.997 & 1.998 & Incremento $\%$ (*) $^{*}$ \\
\hline A CORUÑA & 39,54 & 42,22 & 47,15 & 16,15 \\
ALAVA & 62,57 & 66,64 & 69,20 & 9,58 \\
ALBACETE & 72,32 & 74,72 & 82,18 & 11,99 \\
ALICANTE & 57,85 & 61,27 & 62,11 & 6,84 \\
ALMERIA & 48,87 & 51,72 & 56,54 & 13,56 \\
AVILA & 115,68 & 118,84 & 120,53 & 4,03 \\
BADAJOZ & 51,38 & 52,23 & 53,05 & 3,15 \\
BALEARES & 80,58 & 84,08 & 89,49 & 9,96 \\
BARCELONA & 83,75 & 91,76 & 94,74 & 11,59 \\
BURGOS & 84,27 & 90,61 & 94,63 & 10,94 \\
CACERES & 58,23 & 61,48 & 59,59 & 2,28 \\
CADIZ & 42,21 & 46,17 & 47,62 & 11,35 \\
CASTELLON & 68,00 & 75,17 & 73,24 & 7,15 \\
CEUTA & 73,31 & 76,39 & 76,03 & 3,57 \\
CIUDAD REAL & 60,38 & 63,71 & 63,56 & 4,99 \\
CORDOBA & 67,14 & 70,22 & 74,26 & 9,58 \\
CUENCA & 70,63 & 71,43 & 71,62 & 1,38 \\
\hline
\end{tabular}


Tabla 1 (continuación)

Consumo de carbonato de litio en DHD $\left(^{*}\right)$ por provincias y año en España (1.996-1.998)

\begin{tabular}{|c|c|c|c|c|}
\hline PROVINCIA & 1.996 & 1.997 & 1.998 & Incremento $\%(* *)$ \\
\hline GIRONA & 93,03 & 103,66 & 107,70 & 13,62 \\
\hline GRANADA & 52,08 & 55,97 & 60,92 & 14,52 \\
\hline GUADALAJARA & 87,11 & 88,54 & 99,94 & 12,84 \\
\hline GUIPUZCOA & 73,52 & 94,16 & 77,48 & 5,11 \\
\hline HUELVA & 35,66 & 36,61 & 39,00 & 8,57 \\
\hline HUESCA & 92,20 & 98,82 & 96,72 & 4,67 \\
\hline JAEN & 49,19 & 51,92 & 53,86 & 8,66 \\
\hline LAS PALMAS & 41,25 & 43,39 & 46,24 & 10,78 \\
\hline LEON & 57,43 & 63,04 & 68,58 & 16,26 \\
\hline LOGROÑO & 86,44 & 97,11 & 97,96 & 11,76 \\
\hline LUGO & 58,72 & 68,13 & 75,10 & 21,81 \\
\hline LLEIDA & 84,20 & 98,03 & 103,59 & 18,72 \\
\hline MADRID & 58,13 & 60,37 & 62,40 & 6,84 \\
\hline MALAGA & 41,86 & 45,25 & 44,99 & 6,96 \\
\hline MELILLA & 44,11 & 44,11 & 45,78 & 3,65 \\
\hline MURCIA & 74,46 & 85,56 & 86,37 & 13,78 \\
\hline NAVARRA & 99,73 & 104,92 & 101,65 & 1,88 \\
\hline OURENSE & 48,14 & 49,83 & 53,20 & 9,51 \\
\hline OVIEDO & 58,12 & 60,04 & 59,82 & 2,85 \\
\hline PALENCIA & 76,14 & 84,83 & 89,86 & 15,27 \\
\hline PONTEVEDRA & 40,89 & 45,60 & 51,50 & 20,59 \\
\hline SALAMANCA & 86,11 & 84,98 & 87,91 & 2,05 \\
\hline SANTANDER & 54,14 & 61,32 & 59,66 & 9,26 \\
\hline SEGOVIA & 101,13 & 106,10 & 97,25 & $-3,99$ \\
\hline SEVILLA & 41,07 & 42,30 & 44,60 & 7,91 \\
\hline SORIA & 121,38 & 125,27 & 137,08 & 11,45 \\
\hline STA CRUZ TENERIFE & 56,78 & 61,02 & 66,33 & 14,39 \\
\hline TARRAGONA & 65,73 & 72,72 & 79,66 & 17,49 \\
\hline TERUEL & 67,49 & 71,00 & 69,11 & 2,35 \\
\hline TOLEDO & 71,33 & 81,69 & 88,11 & 19,04 \\
\hline VALENCIA & 77,96 & 80,15 & 82,99 & 6,06 \\
\hline VALLADOLID & 41,64 & 43,64 & 44,81 & 7,09 \\
\hline VIZCAYA & 66,57 & 73,71 & 77,60 & 14,22 \\
\hline ZAMORA & 53,54 & 57,80 & 56,30 & 4,90 \\
\hline ZARAGOZA & 67,19 & 71,58 & 72,73 & 7,62 \\
\hline NACIONAL & 63,36 & 68,08 & 70,28 & 9,84 \\
\hline
\end{tabular}

(*) DHD: Dosis Habitante Día (Casos o DDD/ 100.000 habitantes/ día).

(**) Incremento 1.996-1.998 (\%): Incremento porcentual entre 1.996 a 1.998. 
En general, el consumo de carbonato de litio se mantiene e incrementa en la mayoría de las provincias, con incrementos en torno al 4 y el $10 \%$, si bien hay máximos como Lugo, Pontevedra y Toledo con incrementos porcentuales del 20\%. La tabla 2 presenta el consumo de carbonato de litio en DHD (Dosis
Diaria Definida/ 100.000 habitantes/Día) en el período 1996-1998 en España por comunidades autónomas y año, con diferencias entre ellas. Las mayores cifras corresponden a Navarra, La Rioja y Cataluña; y las más bajas a Andalucía, Galicia y Extremadura.

Tabla 2

Consumo de carbonato de litio en DHD (*) por comunidades autónomas y año en España (1996-1998)

\begin{tabular}{|lcccc|}
\hline COMUNIDAD AUTONOMA & 1.996 & 1.997 & 1.998 & Incremento \%(*) \\
\hline ANDALUCIA & & & 51,38 & 9,92 \\
ARAGÓN & 46,28 & 49,03 & 76,49 & 6,42 \\
ASTURIAS & 71,58 & 76,26 & 59,82 & 2,85 \\
BALEARES & 58,12 & 60,04 & 89,49 & 9,96 \\
CANARIAS & 80,58 & 84,08 & 55,90 & 12,84 \\
CANTABRIA & 48,72 & 51,87 & 59,66 & 9,26 \\
CASTILLA-LA MANCHA & 54,14 & 61,32 & 79,15 & 11,75 \\
CASTILLA Y LEÓN & 69,85 & 74,62 & 78,54 & 8,29 \\
CATALUÑA & 72,02 & 50,95 & 94,96 & 12,72 \\
COMUNIDAD VALENCIANA & 82,89 & 91,36 & 74,69 & 6,41 \\
EXTREMADURA & 69,91 & 73,09 & 55,57 & 2,78 \\
GALICIA & 54,03 & 55,81 & 53,14 & 17,82 \\
MADRID & 43,67 & 47,81 & 62,40 & 6,84 \\
MURCIA & 58,13 & 60,37 & 86,37 & 13,78 \\
NAVARRA & 74,46 & 85,56 & 101,65 & 1,88 \\
PAIS VASCO & 99,73 & 104,92 & 76,43 & 10,68 \\
RIOJA & 68,27 & 79,35 & 97,96 & 11,76 \\
CEUTA & 86,44 & 97,11 & 76,03 & 3,57 \\
MELILLA & 73,31 & 76,39 & 45,78 & 3,65 \\
\hline NATOTAL & 44,11 & 44,11 & 70,28 & 9,84 \\
\hline
\end{tabular}

(*) DHD: Dosis Habitante Día (Casos o DDD/ 100.000 habitantes/ día)

(**) Incremento 1.996-1.998 (\%): Incremento porcentual entre 1.996 a 1.998 


\section{DISCUSIÓN}

Mediante la determinación de las DDD y las DHD, recomendadas por la Nordic Council on Medicines, la Drug Utilization Research Group y la OMS ${ }^{15,16}$, aplicada a la prescripción de carbonato de litio, se estima que la prevalencia de los TB tipo I en España para el período 1996-1998, es de 70 casos por 100.000 habitantes; éstos pueden ser interpretados como que de cada 100.000 habitantes utilizan 70 DDD de carbonato de litio al día, es decir la prevalencia de la enfermedad, o bien que 70 habitantes de cada 100.000 fueron tratados con la DDD del carbonato de litio en España ${ }^{18}$. La cifra calculada es inferior a la obtenida en Noruega con 115 casos por 100.000 habitantes ${ }^{11}$, Australia con 120 por 100.000 habitantes $^{24}$, Suecia con 150 por 100.000 habitantes $^{25}$, y entre 130 y 160 por 100.000 habitantes en el Reino Unido ${ }^{26}$. No obstante, el consumo de carbonato de litio se va adecuando en España a la prevalencia puntual de 80 por 100.000 habitantes del estudio poblacional de Cantabria ${ }^{1,2}$, siendo similar a las cifras de Escocia ${ }^{26}$. Si bien la comparabilidad de los datos es peligrosa por las diferentes metodologías utilizadas en cada cstudio, podemos situar a España en una zona de bajo-medio riesgo ${ }^{27}$.

El consumo de carbonato de litio va creciendo en España debido a la modificación de la pirámide poblacional, con un incremento del estrato de edad en el que aparecen estos trastornos ${ }^{13}$, el aumento de población protegida por el Sistema Nacional de Salud ${ }^{3,4,8,11,14,21}$, el aumento de la esperanza de vida, el mayor acceso a los servicios médicos especializados, la mejora de la calidad de la prescripción en psicofarmacología, el tratamiento específico con carbonato de litio y mayores dosis de mantenimiento ${ }^{49,13,20,24,27}$.

En la tabla 1 se constata un incremento en el consumo de carbonato de litio desde 1996 a 1998 para todas las provincias, excepto para Segovia que parte de cifras ide prevalencia aceptables, que no parece corte- lacionarse con un incremento de la incidencia de la enfermedad sino, mas bien, con una mejora diagnóstica y asistencial de los enfermos, así como a una búsqueda activa de casos en muchas provincias ${ }^{13}$, como ya ha sido descrito en el caso de la Enfermedad de Parkinson ${ }^{21,24}$. En general, es un fármaco que en el período 1985-1994 ha sufrido un incremento del $152 \%$ en España, solo superado por la clorimipramina ${ }^{11}$.

La disponibilidad de los datos de consumo de carbonato de litio en el sistema público, durante el período 1996-1998, es exhaustiva y su uso para estimar la prevalencia de TB está supeditada a que todos los enfermos de TB sean tratados con carbonato de litio, lo que ocurre en el 75-80\% de los casos 8 , a que éstos cumplan con las dosis terapéuticas, ya que los porcentajes de incumplimiento son del 18 al $53 \%$ de los casos 8 y, por otro lado, a que la población enferma acuda al sistema público $0^{4,9,11,13,18-21}$.

Las prescripciones de carbonato de litio para otras patologías han sido confirmadas, pero su uso y la baja prevalencia de estas patologías hacen que el carbonato de litio esté asociado casi exclusivamente con los $\mathrm{TB}^{2,4-8,27}$. La aplicación de este modelo sólo llega a ser estable cuando los datos de ventas se han estabilizado en el tiempo y ha sido completamente introducido el fármaco $0^{9,11,21}$, sin embargo, utilizando este método y comparando los datos con estudios simultáneos de campo, las desviaciones son sólo del $8 \%$ en algunos estudios, aunque en otros las diferencias pueden ser mayores y llegar al $35 \%$, siendo ésta una metodología que subestima la prevalencia real ${ }^{9,11,20,21}$.

Aunque las diferencias observadas en la tabla 1 sobre datos de prevalencia por provincias, podría inducir a pensar en factores de riesgo de exposición diferente para explicarlas 0 , incluso, que pudiera deberse a diferencias en el uso de los servicios médicos o diferencias socioculturales, esto no es así, debido a que existen diferentes pirámides de población en las provincias y las cifras que 
se han estudiado no se han estandarizado. Así, vemos que una de las provincias con mayor prevalencia es Soria ( 1.371 casos/ 100.000 habitantes/ día en 1.998), que es precisamente una de las provincias más envejecidas de España por lo que su prevalencia puede estar relacionada con una mayor supervivencia de estas personas ${ }^{9,13,21}$.

El Sistema Nacional de Salud, dada su amplia cobertura y control exhaustivo de la prescripción y dispensación de medicamentos, reúne condiciones para la determinación de los consumos de DHD de los diferentes principios activos ${ }^{13}$, 14 . En el caso de los TB, la especificidad de la prescripción del carbonato de litio permite deducir la prevalencia de la enfermedad. La validez de los datos viene avalada por el control de estos enfermos por sus especialistas, el consenso en el tratamiento con DDD estándares y por el uso del carbonato de litio casi exclusivamente en esta patología $4,5,7,9,11,18$. Los datos que se manejan en este trabajo incluyen sólo el consumo extrahospitalario a través del Sistema Nacional de Salud, por tanto el consumo real de carbonato de litio en España es necesariamente superior al obtenido ${ }^{20}$. No obstante, dada la cobertura universal y la reforma de la asistencia psiquiátrica emprendida en los años ochenta en España, que ha permitido un mayor acercamiento al ciudadano, por lo que es esperable que las diferencias entre lo hallado y lo real sea pequeña. La propia reforma de la asistencia psiquiátrica ha podido contribuir, en alguna medida, a la tendencia creciente observada en el consumo de carbonato de litio, entre otras razones porque es muy probable que haya derivado pacientes de la asistencia psiquiátrica privada a la pública ${ }^{11,14}$.

Este tipo de estudios de utilización o consumo de medicamentos constituyen un importante medio para la evaluación de la prescripción farmacológica, el conocimiento de las enfermedades y la generación de hipótesis, además de como marcadores simples y eficientes para estimar la prevalencia de enfermedades relacionadas con los fár- macos estudiados; pero se insiste en la necesidad de tener prudencia para estimar la prevalencia a partir de los datos de consumo de fármacos ${ }^{4,9,13}$.

\section{AGRADECIMIENTOS}

A la Subdirección General de Ordenación y Asistencia Farmacéutica del Ministerio de Sanidad y Consumo, el poner a nuestra disposición los datos primarios para la elaboración de este trabajo.

\section{BIBLIOGRAFÍA}

1. Vázquez-Barquero JL, Díez JF, Peña C, Arenal A, Arias M. Estudio comunitario de salud mental de Cantabria: una recopilación final de sus resultados. En: González de la Rivera y Revuelta JL, Rodríguez Pulido F, Sierra López A. El método epidemiológico en salud mental. Barcelona: Masson- Salvat Medicina;1993.p. 165-185.

2. Vázquez-Barquero JL, Díez Manrique JF, Peña C, Quintanal G, López CM. Two stage design in a community survey. Br J Psychiatry 1986; 149: 88-97.

3. García Ruiz AJ, García Ruiz I, Aranda Lara P, Montesinos AC, Martos Crespo F, Sánchez de la Cuesta F. Estudio sobre los enfermos hipertensos en tratamiento en España durante el período 1990-1993. Rev Esp Salud Pública 1996; 71: 9-17.

4. Provencio RM. Estudios de utilización de medicamentos. Rev Neurol (Barc) 1996; 24: 397-399.

5. Johnson RE, Mac Farland BH. Treated prevalences rates of severe mental illness among HMO members. Hosp Comm Psychiatry 1994; 45: 919-924.

6. Price LH, Heninger GR. Lithium in the treatment of mood disorders. N Engl J Med 1994; 331: 591-598.

7. Becerra Fernández A. Acciones biomédicas del Litio: Controversias actuales y posibilidades terapéuticas. Med Clin (Barc) 1994; 103 : 708-714. 
8. Consensus development panel. Mood disorders: Pharmacologic prevention of recurrences. Am J Psychiatry 1985; 142: 469-476.

9. Llop C, Ribas J, Rovira E. Estimación de la prevalencia de depresión a partir de la dosis diaria definida de antidepresivos. Med Clin (Barc) 1998; 110: 557-558.

10. Consejo General de Colegios Oficiales de Farmacéuticos. Catálogo de especialidades Farmacéuticas, 1995 y 1996. Madrid: Consejo General de Colegios Oficiales de Farmacéuticos; $199 j$ y 1996.

11. Alonso MP, de Abajo FJ, Martínez JJ, Montero D, Martín-Serrano G, Madurga M. Evolución del consumo de antidepresivos en España. Impacto de los inhibidores selectivos de la recaptación de serotonina. Med Clin (Barc) 1997; 108: 161-166.

12. Real Decreto $1645 / 1997$, de 31 de octubre del Ministerio de Economía y Hacienda, por el que se declaran oficiales las cifras de población resultantes de la renovación del Padrón Municipal referidas a 1 de mayo de 1996. BOE núm 262, 1 de noviembre de 1997; 31631-31634.

13. Criado Alvarez JJ, Vargas Aragón ML, Romo Barrientos C. Estimación de la prevalencia de trastornos bipolares tipo I en Castilla-La Mancha. Psiquiatría Biológica 1999; 6: 2-6.

14. Fernández Liria A, Diéguez Porres M. La reforma psiquiátrica en España. Aspectos diferenciales. Rev Admon Sanit 1998; 2: 85-102.

15. Nordic Council on Medicines. ATC Index with DDDs. WHO Colaborating Center for Drug Statistics Methodology. Oslo: WHO, 1999.

16. Orden Ministerial de 13 de octubre de 1989, por la que se establece la clasificación anatómica de medicamentos oficial de España (BOE n 257 de 26 de octubre de 1989).

17. Sartor F, Walckiers D. Estimate of disease prevalence using drug consumption data. Am J Epidemiol 1995; 141: 782-787.

18. Los estudios de utilización de medicamentos. En: LH Martín Arias, A Carvajal García-
Pando, El consumo de medicamentos en España. Valladolid: Secretariado de Publicaciones de la Universidad de Valladolid; 1994.

19. García Iñesta A. Estudios de utilización de medicamentos en España y análisis de la situación farmacoterapéutica. En: Instituto Nacional de la Salud, editor. Estudios de utilización de medicamentos. Madrid: Insalud; 1992.p. 15-48.

20. Prieto M, de Abajo FJ, Montero D, MartínSerrano G, Madurga M, Palop R. Uso de antihipertensivos en España, 1985-1995. Med Clin (Barc) 1998; 110: 247-253.

21. Criado Alvarez JJ, Romo Barrientos C, Martínez Hernández J, González Solana I. Consumo de antiparkinsonianos en CastillaLa Mancha. Estimación de la prevalencia de la Enfermedad de Parkinson. Rev Neurol (Barc) 1998; 27: 405-408.

22. Artázcoz Sanz MT, Viñes Rueda JJ. Estimación de la prevalencia de la enfermedad de Parkinson en Navarra. Estudio farmacoepidemiológico del consumo de antiparkinsonianos. Rev Esp Salud Pública 1995; 69: 479-485.

23. Carvajal A, García del Pozo J, Martín Arias LH, Martín de Diego I, Rueda AM, CaroPatón $T$ et al. Cambios en el patrón de consumo de analgésicos opioides en España. Med Clin (Barc) 1997; 109: 281-283.

24. Jacobson GA, Friesen WT, Peterson GM, Rumble RH, Polack AE. Psychoactive drug prescribing in the Tasmanian community. Med J Aust 1992; 157: 20-24.

25. Vestergaard $P$, Schou P. Lithium treatment in Aarhus (1): Prevalence. Pharmacopsychiat 1989; 22: 99-100.

26. Kehoe RF, Mander AJ. Lithium treatment: prescribing and monitoring habits in hospital and general practice. BMJ 1992; 304: 552-554.

27. Ohayon MM, Caulet M, Priest RG, Guilleminault C. Psychotropic medication consumption patterns in the UK general population. J Clin Epidemiol 1998; 51: 273-283. 